The following theorem is an immediate consequence of (4.1) and (2.6):

(4.2) The class of cyclic strongly arcwise connected continua consists exactly of all cyclic locally connected continua $A$ such that every arcpreserving transformation $T(A)=B$, where $B$ is not an arc, is topological.

BROWN UNIVERSITY AND

University of California at Los Angeles

\title{
A NOTE ON SUBGEOMETRIES OF PROJECTIVE GEOMETRY AS THE THEORIES OF TENSORS ${ }^{1}$
}

T. L. WADE

Klein's viewpoint (A) of a geometry as the invariant theory of a transformation group, as formulated in the Erlanger Programm in $1870,{ }^{2}$ has played an important part in the study of geometry during the past half century. A number of explicit utilizations of this viewpoint in invariant aspects of algebraic geometry have been made. ${ }^{3}$ In the last decade the viewpoint (B) of a geometry as the theory of a tensor has received considerable theoretical discussion and utilization in connection with the new differential geometries. ${ }^{4}$ While the adjunction argument, whereby subgeometries of projective geometry result from the latter by holding certain forms latent, has had considerable use, ${ }^{5}$ and is closely related to tensor algebra, there seems to have been no explicit treatment of algebraic invariants for subgeometries of projective geometry from the viewpoint (B) with the use of tensor algebra. To indicate how this might be done is the purpose of this paper. The material here is largely an application and continuation of the basic paper by Cramlet. ${ }^{6}$

\footnotetext{
${ }^{1}$ Presented to the Society, April 27, 1940.

${ }^{2}$ F. Klein, Gesammelte Mathematische Abhandlungen, Berlin, 1921, vol. 1, p. 460.

${ }^{3}$ C. C. MacDuffee, Euclidean invariants of second degree curves, American Mathematical Monthly, vol. 33 (1926), pp. 243-252; Covariants of r-parameter groups, Transactions of this Society, vol. 39 (1933).

${ }^{4}$ J. A. Schouten and J. Haantjes, On the theory of the geometric object, Proceedings of the London Mathematical Society, vol. 42 (1937), pp. 356-376.

${ }^{5} \mathrm{H}$. Weyl, The Classical Groups: Their Invariants and Representations, Princeton University Press, 1939, pp. 254-258; H. W. Turnbull, The Theory of Determinants, Matrices, and Invariants, Blackie and Son, 1929, chap. 21.

${ }^{6} \mathrm{C}$. M. Cramlet, The derivation of algebraic invariants by tensor algebra, this Bulletin, vol. 34 (1928), pp. 334-342.
} 
If in the $(n-1)$ projective geometry we hold the linear form $L_{i} X^{i}$, where $L_{i}=(0, \cdots, 0,1)$ latent, we have the special numerical covariant tensor $L_{i}$. Then from Theorems 1 and 2 of Cramlet we have the following theorem:

THEOREM 1. Affine geometry as a subgeometry of projective geometry is the theory of the tensor $L_{i}$.

Similarly, $U_{i}$ being dual covariant coordinates, holding the quadratic form

$$
E^{i j} U_{i} U_{j}=0
$$

latent, where

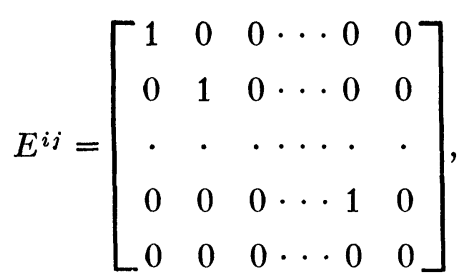

we have a second theorem:

THEOREM 2. Euclidean geometry as a subgeometry of affine geometry is the theory of the tensor $E^{i j}$, and as a subgeometry of projective geometry is the theory of the tensors $L_{i}$ and $E^{i j}$.

THEOREM 3. Every euclidean concomitant for a set of ground forms in $X^{i}$ and dual variables $U_{i}$, where $X^{i} U_{i}=0$, is expressible by composition as a tensor of order zero with the use of the coefficient tensors of the ground forms and the tensors $\epsilon^{i_{1} \cdots i_{n}}, \epsilon_{i_{1}} \cdots i_{n}, L_{i}, E^{i j}, X^{i}$, and $U_{i}$. The first two of these tensors are the commonly used skew symmetrical numerical tensors.

In applications it is advantageous to in troduce the tensor $A^{i_{1} \cdots i_{n-1}}$ where $A^{i_{1} \ldots i_{n-1}}=\epsilon^{i_{1} \ldots i_{n}} L_{i_{n}}$. For $n=3$,

$$
A^{i j}=\epsilon^{i j k} L_{k}=\left[\begin{array}{rrr}
0 & 1 & 0 \\
-1 & 0 & 0 \\
0 & 0 & 0
\end{array}\right] \text {. }
$$

As an illustration of an application we give a corallary:

COROLlary. An algebraically complete system of euclidean invariants for the ternary cubic curve 


$$
C_{i j k} X^{i} X^{j} X^{k}=0, \quad i, j, k=1,2,3 ; C_{i j k}=C_{i k j}=C_{j i k},
$$

consists of the seven invariants

$$
\begin{aligned}
& I_{1}=C_{a_{1} b_{1} c_{1}} C_{a_{2} b_{2} c_{2}} A^{a_{1} a_{2}} A^{b_{1} b_{2}} E^{c_{1} c_{2}}, \\
& I_{2}=C_{a_{1} a_{2} c_{1}} C_{b_{1} b_{2} c_{2}} E^{a_{1} a_{2}} E^{b_{1} b_{2}} E^{c_{1} c_{2}} \text {, } \\
& I_{3}=C_{a_{1} b_{1} c_{1}} C_{a_{2} b_{2} d_{1}} C_{a_{3} c_{2} d_{2}} \epsilon^{a_{1} a_{2} a_{3}} A^{b_{1} b_{2}} A^{c_{1} c_{2}} A^{d_{1} d_{2}}, \\
& I_{4}=C_{a_{1} b_{1} e_{1}} C_{a_{2} b_{2} f_{1}} C_{c_{1} d_{1} e_{2}} C_{c_{2} d_{2} f_{2}} A^{a_{1} a_{2}} A^{b_{1} b_{2}} A^{c_{1} c_{2}} A^{d_{1} d_{2}} A^{e_{1} e_{2}} A^{f_{1} f_{2}} \text {, } \\
& I_{5}=C_{a_{1} b_{1} c_{1}} C_{b_{2} c_{2} d_{1}} C_{c_{3} d_{2} a_{2}} C_{d_{3} a_{3} b_{3}} \epsilon^{a_{1} a_{2} a_{3}} \epsilon^{b_{1} b_{2} b_{3}} \epsilon^{c_{1} c_{2} c_{3}} \epsilon^{d_{1} d_{2} d_{3}}, \\
& I_{6}=C_{a_{1} b_{1} c_{1}} C_{a_{2} b_{2} d_{1}} C_{b_{3} c_{2} e_{1}} C_{c_{3} a_{3} f_{1}} C_{d_{2} e_{2} f_{2}} C_{d_{3} e_{3} f_{3}} \\
& \cdot \epsilon^{a_{1} a_{2} a_{3}} \epsilon^{b_{1} b_{2} b_{3}} \epsilon^{c_{1} c_{2} c_{3}} \epsilon^{d_{1} d_{2} d_{3}} \epsilon^{e_{1} e_{2} e_{3}} \epsilon^{f_{1} f_{2} f_{3}} \\
& I_{7}=C_{a_{1} b_{1} c_{1}} C_{d_{1} e_{1} f_{1}} A^{a_{1} d_{1}} \epsilon^{b_{1} e_{1} \sigma_{1}} E^{c_{1} f_{1}} \\
& \text { - } C_{a_{2} b_{2} c_{2}} C_{d_{2} e_{2} f_{2}} A^{a_{2} d_{2}} \epsilon^{b_{2} e_{2} g_{2}} E^{c_{2} f_{2}}
\end{aligned}
$$

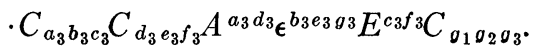

In expanded form

$$
\begin{gathered}
I_{1}=2\left[C_{111} C_{122}-\left(C_{112}\right)^{2}-\left(C_{122}\right)^{2}+C_{112} C_{222}\right], \\
I_{2}=\left(C_{111}+C_{122}\right)^{2}+\left(C_{112}+C_{222}\right)^{2}, \\
I_{3}=6\left|\begin{array}{lll}
C_{111} & C_{112} & C_{113} \\
C_{211} & C_{221} & C_{231} \\
C_{221} & C_{222} & C_{223}
\end{array}\right| .
\end{gathered}
$$

The expansions of the other invariants are considerably longer; $I_{5}$ contains twenty-five terms and $I_{6}$ one hundred and three terms. These seven invariants are, except in some cases for a constant factor, the members of the algebraically complete system of euclidean invariants for the cubic curve found through geometric means by Thomae. ${ }^{7}$

An invariant study of the geometry associated with the GalileiNewton group of ten parameters has been made by Weitzenböck using the symbolic notation. ${ }^{8}$ In the Galilei-Newton geometry, with $X^{i}$ $(i=1, \cdots, 5)$ the homogeneous coordinates of a point, the basic latent forms may be represented by

$$
K_{i} X^{i}
$$

where $K_{i}=(0,0,0,0,1)$;

${ }^{7} \mathrm{~J}$. Thomae, Ueber orthogonale Invarianten der Curven dritter Ordnung, Berichte der Saechsischen Akademie der Wissenschaften, Leipzig, vol. 51 (1899), pp. 317-353.

${ }^{8} \mathrm{R}$. Weitzenböck, Die Invarianten der Galilei-Nerwton-Gruppe, Mathematische Annalen, vol. 80 (1920). 


$$
L_{i} X^{i}
$$

where $L_{i}=(0,0,0,1,0)$;

$$
Q^{i j} U_{i} U_{j}
$$

where

$$
Q^{i j}=\left[\begin{array}{lllll}
1 & 0 & 0 & 0 & 0 \\
0 & 1 & 0 & 0 & 0 \\
0 & 0 & 1 & 0 & 0 \\
0 & 0 & 0 & 0 & 0 \\
0 & 0 & 0 & 0 & 0
\end{array}\right]
$$

Here $X^{i} U_{i}=0$.

From this approach we are led to the following theorem:

THEOREM 4. Every concomitant in Galilei-Newton geometry for a set of ground forms in $X^{i}$ and dual variables $U_{i}$ is expressible by composition as a tensor of order zero with the coefficient tensors of the ground forms and the tensors

$$
\epsilon^{i_{1} \cdots i_{5}}, \epsilon_{i_{1} \cdots i_{5}}, K_{i}, L_{i}, Q^{i j}, X^{i}, U_{i} .
$$

This theorem, in directness of approach and in simplicity of application, seems to have advantages over the concluding statement of Weitzenböck, which concerns itself with twelve basic symbolic factors for the Galilei-Newton group.

University of Alabama 